\title{
Determinants and outcomes of change in physical activity in COPD
}

\author{
Ana Kantorowski ${ }^{1}$, Emily S. Wan ${ }^{1,2,3,4}$, Diana Homsy ${ }^{1}$, Reema Kadri ${ }^{5}$, \\ Caroline R. Richardson ${ }^{5}$ and Marilyn L. Moy (1) ${ }^{1,2,4}$
}

Affiliations: ${ }^{1}$ Pulmonary and Critical Care Medicine Section, VA Boston Healthcare System, Boston, MA USA. ${ }^{2}$ Dept of Veterans Affairs, Rehabilitation Research and Development Service, Washington, DC, USA ${ }^{3}$ Channing Division of Network Medicine, Brigham and Women's Hospital, Boston, MA, USA. ${ }^{4}$ Harvard Medical School, Boston, MA, USA. ${ }^{5}$ Dept of Family Medicine, University of Michigan, Ann Arbor, MI, USA.

Correspondence: Marilyn L. Moy, VA Boston Healthcare System, Pulmonary and Critical Care Section, 1400 VFW Parkway, Mail Code 111PI, Boston, MA 02132, USA. E-mail: marilyn.moydava.gov

ABSTRACT Determinants of change in physical activity and outcomes of physical activity promotion are unclear. In this secondary analysis of a randomised controlled trial of a physical activity intervention, we assess predictors of change in physical activity and the effects of increasing physical activity on chronic obstructive pulmonary disease (COPD) measures.

Physical activity was promoted in 94 subjects with COPD using the Omron HJ-720ITC pedometer alone or the pedometer plus a website that provides goal setting, feedback, motivational and educational messages, and social support for 3 months. We assessed forced expiratory volume in $1 \mathrm{~s}$ (FEV1), 6-min walk test (6MWT) distance, depression, social support and markers of systemic inflammation (C-reactive protein (CRP) and interleukin (IL)-6). Data from both groups were combined and subjects categorised as responders (increased steps per day) or nonresponders (decreased steps per day). Linear regression models explored predictors of change in physical activity and assessed the effect of response on changes in COPD measures.

The cohort of responders $(n=62)$ and nonresponders $(n=32)$ had mean FEV1 $1.89 \pm 0.64 \mathrm{~L}(63 \pm 22 \%$ predicted). Baseline steps per day, diagnosis of depression, social support, oxygen use and season significantly predicted change in daily step count. Responders had increases in physical activity (2038 steps per day), FEV1 $(308 \mathrm{~mL})$ and 6MWT distance $(43.6 \mathrm{~m})$, and decreases in CRP $\left(7.84 \mathrm{mg} \cdot \mathrm{L}^{-1}\right)$ and IL-6 $\left(2.73 \mathrm{ng} \cdot \mathrm{mL}^{-1}\right)$ compared with nonresponders $(\mathrm{p}<0.0001-0.009)$.

History of depression, social support, oxygen use and season predict change in physical activity, and should be routinely assessed in exercise counselling. Increases in physical activity are associated with improvements in lung function, exercise capacity and systemic inflammation.

@ERSpublications

History of depression, social support, oxygen use and season predict change in physical activity in COPD. Response to physical activity promotion is associated with improvements in lung function, exercise capacity and systemic inflammation. http://ow.ly/oOMA30kPGCE

Cite this article as: Kantorowski A, Wan ES, Homsy D, et al. Determinants and outcomes of change in physical activity in COPD. ERJ Open Res 2018; 4: 00054-2018 [https://doi.org/10.1183/ 23120541.00054-2018].

This article has supplementary material available from openres.ersjournals.com

Received: April 132018 | Accepted after revision: July 052018

The content of this work is not subject to copyright. Design and branding are copyright @ERS 2018. This article is open access and distributed under the terms of the Creative Commons Attribution Non-Commercial Licence 4.0. 


\section{Introduction}

The Global Initiative for Chronic Obstructive Lung Disease guidelines recommend regular physical activity for all persons with chronic obstructive pulmonary disease (COPD) [1]. Observational cross-sectional [2-6] and longitudinal [7, 8] studies have demonstrated associations between higher levels of physical activity and better COPD health outcomes, such as decreased risk of acute exacerbations, hospital (re)admissions and death, independent of forced expiratory volume in $1 \mathrm{~s}$ (FEV1), and identified predictors of physical activity [9]. Although a number of interventions have been shown to be efficacious to increase physical activity in persons with COPD, knowledge gaps about change in physical activity exist [10-15]. Determinants of change in physical activity, as a result of active physical activity promotion, are unclear. The relationships between increases in physical activity, in response to physical activity promotion, and COPD health measures are not well understood.

Studies to date have focused on testing the efficacy of specific physical activity interventions [10-15]. Identification of characteristics that predict change in physical activity, independent of specific physical activity interventions, would allow targeted selection of patients who would benefit from physical activity promotion. Attention to such characteristics would also optimise patient responses to physical activity promotion. In addition, the summary group values for change in physical activity reported in these studies do not capture the heterogeneity of individual responses [10-15]. Not all participants in intervention groups have the same degree of improvement and subsets of participants in control groups increase physical activity [10-15]. We assert that we can enhance our understanding of the effects of increasing physical activity on COPD measures by examining individual responses to physical activity promotion rather than limiting assessments to randomised group responses to specific physical activity interventions.

We developed a physical activity intervention based on the Behavioural Theory of Self-Regulation that combines the Omron HJ-720ITC pedometer (Omron Healthcare, Bannockburn, IL, USA) and a website that provides goal setting, feedback, motivational and educational messages, and social support [12, 14, 16-18]. We have previously demonstrated its efficacy to increase daily step counts in two randomised controlled trials (RCTs) $[12,14]$. In one study, Every Step Counts (ESC), physical activity was promoted with a pedometer alone or with a pedometer plus website for 3 months, and participants were well characterised at baseline and end of study [14]. In this secondary analysis, we combined the two physical activity promotion groups to 1) explore physiological and psychosocial characteristics that predict change in daily step counts, and 2) examine the effect of increases in physical activity on changes in COPD measures.

\section{Methods}

This is a secondary analysis of data from the ESC study (ClinicalTrials.gov identifier NCT01772082) [14] The design was a single-site RCT which compared a pedometer plus website that provided goal setting, feedback, motivational and educational content, and social support to a pedometer alone. A previous publication, which adhered to the CONSORT checklist for reporting randomised trials, described the research methods in detail [14]. Results for the primary outcome of daily step count have been reported [14]. The protocol was approved by the VA Boston Healthcare System Committee on Human Research (approval 2328) and written informed consent obtained.

\section{Patient recruitment and eligibility}

Eligible participants were enrolled from the pulmonary clinics at VA Boston Healthcare System (Boston, MA, USA) between 2012 and 2016, and were $\geqslant 40$ years of age, had COPD with a smoking history of $\geqslant 10$ pack-years and a $\mathrm{FEV} 1 /$ forced vital capacity ratio $<0.70$ or emphysema on clinical chest computed tomography, and had access to the internet [14]. Exclusion criteria included inability to ambulate without assistance and unstable cardiovascular disease [14].

\section{Physical activity promotion groups}

Participants were randomly assigned in a 1:1 ratio to pedometer alone or pedometer plus website for 3 months [14]. The pedometer-alone group was instructed to wear the Omron pedometer every day and upload step-count data via the website at least monthly. They received written materials about exercise, but no step-count goals or access to content on the website. The pedometer-plus-website group was instructed to wear the pedometer every day and upload at least weekly. They received weekly step-count goals, access to motivational and educational content, and an online community forum [12, 14, 16-18].

\section{Data collection}

Data were collected on participants' characteristics chosen a priori for possible association with physical activity. Participants attended in-person study visits at baseline and end of study. Medical history, comorbidities, medication use, supplemental oxygen use and acute exacerbations prior to study entry were 
assessed. Lung function was measured using an Eaglet spirometer (nSpire Health, Longmont, CO, USA) [19]. The 6-min walk test (6MWT) was performed according to American Thoracic Society guidelines [20]; a practice walk was not completed. C-reactive protein (CRP) and interleukin (IL)-6 were measured using a high-sensitivity immunoturbidimetric assay with a sensitivity of $0.03 \mathrm{mg} \cdot \mathrm{L}^{-1}$ and $0.094 \mathrm{pg} \cdot \mathrm{mL}^{-1}$, respectively (Clinical and Epidemiologic Research Laboratory, Children's Hospital, Boston, MA, USA) [6]. Health-related quality of life (HRQoL) was assessed with the St George's Respiratory Questionnaire-Total Score (SGRQ-TS) [21]. Dyspnoea was assessed with the modified Medical Research Council (mMRC) scale [22]. Diagnosis of depression was self-reported and current depression was assessed with the Beck Depression Inventory (BDI)-II [23]. Social support was assessed with the Medical Outcome Study (MOS) social support survey, with total score, and domains of emotional support, tangible support, affectionate support and positive interaction [24]. Higher scores reflect greater social support. During the study, acute exacerbations, emergency room visits and hospitalisations for pulmonary reasons were tracked.

Omron pedometer accuracy and step-count analyses have been previously detailed [25, 26]. Baseline daily step count was averaged over at least 5 wear-days, collected over 7 days, prior to randomisation. End-of-study step-count data were averaged over at least 5 wear-days collected over 7 consecutive days, between days 77 and 90 after randomisation. A wear-day was defined as one that recorded $\geqslant 100$ steps and $\geqslant 8 \mathrm{~h}$ of wear-time $[12,14,25]$, and was a surrogate measure of Omron adherence. Change in daily step count was the difference between the end-of-study and baseline daily step counts.

Data analysis

Subjects in both physical activity promotion groups were combined and categorised as responders (increased daily step counts at end of study) and nonresponders (decreased). Since we previously showed that the natural history of daily step count in persons with COPD is to decrease over 3 months [27], we assigned the one participant who had no change in daily step count to the responder group. In a sensitivity analysis, we excluded this one participant; the results were unchanged so we present the results with him in the responder group. The unpaired t-test, Wilcoxon rank-sum test or Fisher's exact test were used to compare characteristics at baseline and during the study between responders and nonresponders.

Generalised linear regression models (PROC GLM, SAS version 9.4; SAS Institute, Cary, NC, USA) were used to explore baseline characteristics (table 1), chosen a priori to possibly impact physical activity, as predictors of overall change in daily step count, adjusting for baseline daily step count and physical activity promotion group. Generalised linear regression models were also used to assess the effect of response to physical activity promotion on changes in COPD measures. Multivariate models adjusted for the baseline COPD measure, physical activity promotion group, FEV1 \% pred [28] and possible confounders (variables that were suggested to differ between the responder and nonresponder groups with a permissive threshold of $\mathrm{p}<0.20$ ). For all models, data were checked for linear relationship, absence of collinearity, homoscedasticity and normal distribution of residuals. Statistical significance was defined as $\mathrm{p}<0.05$.

\section{Results}

\section{Subject characteristics}

A total of 111 participants completed the study, with 97 subjects having enough wear-days to calculate an average daily step count at enrolment and end of study. The 14 participants $(n=8$ pedometer alone and $\mathrm{n}=6$ pedometer plus website) who did not have sufficient step-count data were no different from the 97 subjects who did, with respect to baseline daily step count, FEV1 \% pred, 6MWT distance and SGRQ-TS $(\mathrm{p}=0.207-0.559)$. Three subjects with baseline step count exceeding 10000 steps per day were excluded as outliers from the analysis. Thus, 94 subjects were included in the analysis $(n=43$ pedometer alone and $\mathrm{n}=51$ pedometer plus website). The cohort had mean \pm SD age $69 \pm 8$ years and FEV1 $1.89 \pm 0.64 \mathrm{~L}(63 \pm 22 \%$ predicted).

A subgroup of persons in both physical activity promotion groups improved their daily step count. Altogether, there were 62 responders ( $n=27$ pedometer alone and $n=35$ pedometer plus website) and 32 nonresponders $(n=16$ pedometer alone and $n=16$ pedometer plus website). The responders had an average increase in physical activity of $1243 \pm 1405$ steps per day (median interquartile range (IQR)) 708 (228-1753)), while the nonresponders had an average decrease of $1387 \pm 1252$ steps per day (median (IQR) $-1178(-1811--355))$.

Table 1 shows characteristics at baseline and during the study that may differ $(p<0.20)$ between the responders and nonresponders: baseline daily step count, body mass index, current smoker, diagnosis of depression, back pain, MOS social support (total and domain scores), season of enrolment, acute exacerbation during the study, emergency room visit for a pulmonary problem during the study and a pulmonary hospitalisation during the study. Responders had significantly lower baseline daily step count, 
TABLE 1 Subject characteristics

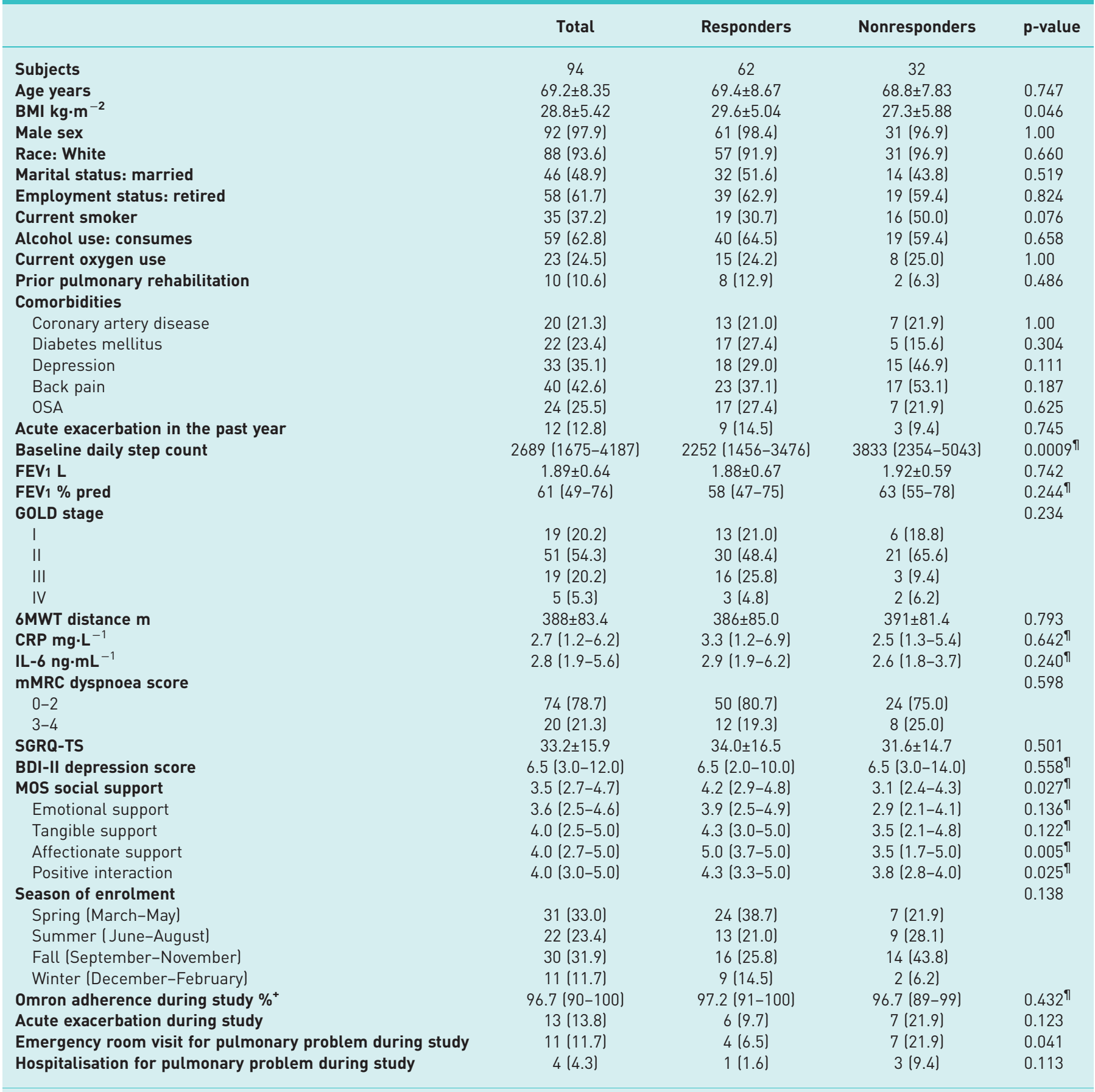

Data are presented as $n$, mean \pm SD or $n(\%)$ for characteristics normally distributed, or median (interquartile range) for characteristics nonnormally distributed, where normal distribution was determined using the Shapiro-Wilk value at the 0.05 level. BMI: body mass index; OSA: obstructive sleep apnoea; FEV1: forced expiratory volume in $1 \mathrm{~s}$; GOLD: Global Initiative for Chronic Obstructive Lung Disease; 6MWT: 6-min walk test; CRP: C-reactive protein; IL: interleukin; mMRC: modified Medical Research Council; SGRQ-TS: St George's Respiratory Questionnaire-Total Score; BDI: Beck Depression Inventory; MOS: Medical Outcomes Study. Fisher's exact tests were used to test for significance among categorical variables. Unpaired t-tests were used for continuous variables unless otherwise indicated. ${ }^{\#}$ : characteristics

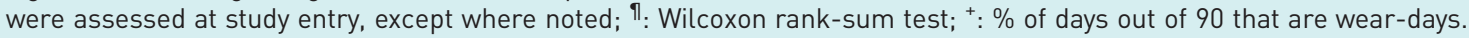

greater social support and fewer emergency room visits for pulmonary problems during the study compared with nonresponders (table 1). There were no significant differences in baseline 6MWT distance, SGRQ-TS, CRP and IL-6, and percentage of participants who had participated in pulmonary rehabilitation or had an acute exacerbation or hospitalisation prior to study entry (table 1). 


\begin{tabular}{|c|c|c|}
\hline Predictors & $\begin{array}{l}\text { Effect on change in daily } \\
\text { step count }{ }^{\#}(95 \% \text { CI) }\end{array}$ & p-value \\
\hline Baseline daily step count (per 1000 steps increase) & $-423(-601--245)$ & $<0.0001$ \\
\hline Diagnosis of depression (Ref.=no diagnosis) & $-865(-1547--182)$ & 0.014 \\
\hline MOS social support & $324(35.3-613)$ & 0.028 \\
\hline Oxygen use (Ref.=no oxygen) & $-803(-1574-31.7)$ & 0.042 \\
\hline \multicolumn{3}{|l|}{ Season of enrolment (Ref.=Spring) } \\
\hline Summer ( June-August) & $-978(-1854--102)$ & 0.029 \\
\hline Fall (September-November) & $-1267(-2060--474)$ & 0.002 \\
\hline Winter (December-February) & $-218(-1338-903)$ & 0.700 \\
\hline \multicolumn{3}{|c|}{$\begin{array}{l}\text { MOS: Medical Outcomes Study; Ref.: reference group. \#: effects on change in daily step count are the } \\
\text { model coefficients as determined by one generalised linear regression model, adjusted for physical } \\
\text { activity promotion group. }\end{array}$} \\
\hline
\end{tabular}

\section{Baseline predictors of change in daily step count}

Baseline daily step count, self-reported diagnosis of depression, social support, oxygen use, and enrolment in Summer and Fall were significant predictors of change in daily step count, independent of physical activity promotion group (see supplementary material and table 2). For each 1000 steps increase in baseline step count, there was an average 423 steps per day decrease in the change in daily step count (95\% CI $-601--245$ steps per day; $\mathrm{p}<0.0001)$. Those with a self-reported diagnosis of depression had an average decrease of 865 steps per day in change in daily step count, compared with those without depression (95\% CI $-1547--182$ steps per day; $\mathrm{p}=0.014$ ). For each unit increase in social support, there was an average 324 steps per day increase in the change in daily step count (95\% CI 35.3-613 steps per day; $\mathrm{p}=0.028$ ). Those using oxygen had an average decrease of 803 steps per day in change in daily step count, compared with those not using oxygen (95\% CI $-1574--31.7$ steps per day; $\mathrm{p}=0.042$ ). Compared with those enrolled in the Spring, those enrolled in the Summer and Fall had significant decreases in change in daily step count (table 2).

\section{Effect of response to physical activity promotion on changes in COPD measures}

Response to physical activity promotion was significantly associated with improvements in COPD measures (see supplementary material and table 3). In adjusted linear regression models, compared with nonresponders, responders to physical activity promotion had a significant increase in daily step count of 2038 steps (95\% CI 1369-2708 steps; $\mathrm{p}<0.0001)$, FEV1 of $308 \mathrm{~mL}$ (95\% CI 78.6-538 mL; p=0.009), 6MWT distance of $43.6 \mathrm{~m}$ (95\% CI 21.8-65.4 m; p=0.0002), and a significant decrease in CRP and IL-6 of $7.84 \mathrm{mg} \cdot \mathrm{L}^{-1}\left(95 \% \mathrm{CI}-12.8--2.89 \mathrm{mg} \cdot \mathrm{L}^{-1} ; \mathrm{p}=0.0023\right)$ and $2.73 \mathrm{ng} \cdot \mathrm{mL}^{-1}\left(95 \% \mathrm{CI}-4.38--1.08 \mathrm{ng} \cdot \mathrm{mL}^{-1}\right.$; $\mathrm{p}=0.002$ ), respectively (table 3 ). There was no significant effect on change in mMRC dyspnoea score, BDI-II depression score or SGRQ-TS.

\section{Discussion}

A diagnosis of depression, less social support, supplemental oxygen use and season predict overall decreases in physical activity, in participants with COPD in whom physical activity was actively promoted. We show that increases in physical activity, independent of the intervention, are associated with improvements in lung function and exercise capacity, and decreases in markers of systemic inflammation. Our findings highlight the complex interactions between psychosocial and physiological factors in physical activity promotion. Psychosocial factors appear important in determining change in physical activity, which in turn have significant effects on physiological measures.

All persons with COPD do not respond to physical activity promotion in the same way. Our results may allow targeted selection of COPD patients who would benefit most from physical activity promotion strategies. Our results also identify characteristics that may inform exercise counselling strategies to optimise individual responses to physical activity promotion. Addressing barriers of using supplemental oxygen and season/temperature changes on engagement in physical activity may increase responses to physical activity interventions. Self-reported diagnosis of depression was predictive of change in daily step count, while the BDI-II score was not. This discrepancy may be attributable to the fact that the BDI-II screens for current depressive symptoms that may be adequately treated in subjects who self-report a diagnosis of depression [23]. There is also an interaction of anxiety, a domain that we did not formally assess in this study, and current depression symptoms [29]. Our results using physical activity change data from physical activity promotion strategies extend previous cross-sectional studies that showed associations 
TABLE 3 Effect of response to physical activity promotion on change in chronic obstructive pulmonary disease (COPD) measures

\begin{tabular}{|c|c|c|}
\hline Change in COPD measure & Effect on change in COPD measure ${ }^{\#}(95 \% \mathrm{CI})$ & p-value \\
\hline Daily step count & 2038 (1369-2708) & $<0.0001$ \\
\hline FEV $1 L^{\top}$ & $0.308(0.0786-0.538)$ & 0.009 \\
\hline FEV $1 \%$ pred & $10.63(2.14-19.1)$ & 0.015 \\
\hline 6MWT distance m & $43.6(21.8-65.4)$ & 0.0002 \\
\hline CRP $\mathrm{mg} \cdot \mathrm{L}^{-1}$ & $-7.84(-12.8--2.89)$ & 0.0023 \\
\hline $\mathrm{IL}-6 \mathrm{ng} \cdot \mathrm{mL}^{-1}$ & $-2.73(-4.38--1.08)$ & 0.002 \\
\hline mMRC dyspnoea score & $0.154(-0.0581-0.365)$ & 0.153 \\
\hline BDI-II depression score & $0.0954(-2.36-2.55)$ & 0.939 \\
\hline SGRQ-TS & $-3.52(-8.79-1.76)$ & 0.188 \\
\hline
\end{tabular}

FEV1: forced expiratory volume in $1 \mathrm{~s}$; 6MWT: 6-min walk test; CRP: C-reactive protein; IL: interleukin; mMRC: modified Medical Research Council; BDI: Beck Depression Inventory; SGRQ-TS: St George's Respiratory Questionnaire-Total Score. * : effects on change in COPD measure are the model coefficients as determined by nine separate generalised linear regression models, adjusted for baseline COPD measure, physical activity promotion group, baseline daily step count, FEV1 \% pred, body mass index, smoking status, history of depression, back pain, Medical Outcomes Study social support, season of enrolment, acute exacerbation during study, emergency room visit for pulmonary problem during study and pulmonary hospitalisation during study; ": model does not include FEV 1 \% pred. Responders/ nonresponders is the independent variable (reference group=nonresponders); change in COPD measure (3-month minus baseline values) is the dependent variable.

between diagnosis of depression and reduced physical activity in COPD [30, 31]. Social support is predictive of change in physical activity, a finding consistent with observations in the general elderly population in whom social isolation is associated with worse functional status [32]. Routine clinical assessment for depression and effects of social support, oxygen use, and season on engagement in physical activity may increase physical activity in persons with COPD.

Our results, based on individual responses to physical activity promotion rather than on randomisation groups for a specific physical activity intervention trial, contribute to our understanding of the effects of physical activity promotion on COPD health measures [9-13]. We show that increases in daily step count are significantly associated with increases in FEV1 and 6MWT distance. The average increase in FEV1 of $308 \mathrm{~mL}$ associated with response to physical activity promotion is of the same magnitude as values reported in COPD pharmacological trials [33, 34]. Our results add to an observational study that followed patients with COPD over 10 years, which showed that increases in physical activity are associated with higher FEV1 [7]. It has been previously shown that a pedometer-based physical activity intervention can increase 6MWT distance by $13.4 \mathrm{~m}$ [13]. The mechanisms by which increases in physical activity might lead to improvement in lung function are unclear. A possible mechanism is that exercise is a natural bronchodilator in humans, with reduction of vagal tone [35]. The observed difference in 6MWT distance of $43.6 \mathrm{~m}$ between responders and nonresponders is within the range of $30-50 \mathrm{~m}$ for the minimal clinically important difference $[36,37]$. In our trial, participants were clinically stable at the time of study entry. The models adjusted for acute exacerbations, emergency room visits and pulmonary hospitalisations during the study. Therefore, it is unlikely that occurrence and treatment of these events explain the observed increases in step count, and improved lung function and exercise capacity.

Our results also begin to elucidate the mechanisms of benefit of increasing physical activity and its effects on COPD outcomes. We observed significant associations between increases in daily step count and decreases in CRP and IL-6. We have previously shown in an observational study that persons with COPD who walk the least have the highest levels of systemic inflammation, independent of lung function, history of acute exacerbations, statin use and cardiovascular disease [6]. Taken together, these results enhance our understanding of markers of systemic inflammation as possible mediators of the observed benefits of physical activity in COPD [38].

Change in daily step count was not associated with significant changes in dyspnoea, HRQoL or BDI-II scores. Physical activity promotion for $>3$ months may be needed to impact these symptoms and psychosocial outcomes. We previously showed that persons with COPD do not walk more due to worries about becoming short of breath, oxygen levels becoming low or the need to use inhalers [39]. Future interventions should comprehensively address these concerns, which may persist despite increases in physical activity and exercise capacity, to ultimately impact perceived dyspnoea, HRQoL and current depression scores. 
We showed that persons with COPD who responded to physical activity promotion walked on average 2038 steps per day more than those who did not respond. This value is greater than the 804 steps per day increase that we previously reported for the ESC RCT, which assessed the additional impact of the website over pedometer alone [14]. The 2038 steps per day most likely reflect that our control group used a pedometer that promoted physical activity as well. Other pedometer-based interventions in COPD have shown similar increases in daily step count of 3000 steps per day [11] and 1469 steps per day [13]. Taken together, it is plausible that increases of 1500-3000 steps per day could impact COPD measures such as $6 \mathrm{MWT}$ distance and markers of systemic inflammation, and lead to observed decreases in risk of acute exacerbations and healthcare utilisation [10].

A major strength of the study is our comprehensive assessment of physiological and psychosocial variables that influence behaviour change and engagement in physical activity. The use of two types of physical activity promotion strategies is a strength. The elderly COPD population will have different preferences for and access to modalities such as pedometers and websites. Our analyses focus on response to physical activity promotion and our results are independent of the specific physical activity intervention.

Our study has several limitations. It is a secondary and exploratory analysis, but provides support for further hypothesis-driven studies. Our qualitative definition of responders and nonresponders does not utilise a clinically important difference in step count. In one published study, the range for the clinically important difference (599-1131 steps per day) from distribution-based methods was wide [40]. We performed a sensitivity analysis using the published cut-offs, but the resulting small sample sizes limited meaningful conclusions. Generalisability may be limited given that most participants had mild-moderate COPD and this is a single-centre study located geographically in the northeast of the USA in a cohort of predominantly White males. Further studies are required to externally validate these results.

We acknowledge that a practice test was not included as part of the 6MWT protocol. When we began enrolment in April 2012, our protocol was consistent with guidelines at the time [20]. When the recommendations for a practice 6MWT were published [41, 42], we felt that changing the protocol mid-study was unwise considering 1) the need to maintain consistency in our data collection methods given our primary interest in change scores, and 2) the potential increase in dropout rate given the added burden to perform the $6 \mathrm{MWT}$ on two separate days. We believe that our $6 \mathrm{MWT}$ values are valid for the following reasons. First, the intra-class correlations of $6 \mathrm{MWT}$ conducted within a short time span (0-10 days) are high (0.82-0.99), so a repeat test performed a day later, at study entry and end of study, would be very similar to the first test $[41,43,44]$. Second, the effects of learning on the second tests are not known to be durable (beyond a few days); it is unlikely that the 6MWT conducted at the end-of-study visit would be impacted by learning effects from the test at study entry conducted 3 months earlier. Third, the difference in $6 \mathrm{MWT}$ distance between the responder and nonresponder groups $(43.6 \mathrm{~m})$ exceeds the increases expected to be attributable to a learning effect (mean $26.1 \mathrm{~m}, 95 \%$ CI 24-29 m) [41, 44]. Finally, since there was no practice test at either time-point, before or after the study period, we believe that the change scores of the 6MWT are unaffected.

History of depression, social support, oxygen use and season are predictors of change in physical activity, and should be routinely assessed in exercise counselling. Increases in physical activity are associated with beneficial changes in lung function, exercise capacity and systemic inflammation. Additional studies are needed to understand causal effects of increasing physical activity and elucidate mechanisms of benefit.

Acknowledgements: We thank the veterans who participated in this study.

Author contributions: M.L. Moy and C.R. Richardson were involved in the conception and design of all stages of the study. A. Kantorowski, D. Homsy and R. Kadri were involved in study data collection. A. Kantorowski and E.S. Wan conducted study analyses. All authors read and approved the final manuscript. M.L. Moy and A. Kantorowski had full access to all the data in the study, and take responsibility for the integrity of the data and the accuracy of the data analysis. The views expressed in this article do not communicate an official position of the Dept of Veterans Affairs.

Conflict of interest: M.L. Moy reports personal fees (honorarium for consultancy) from AstraZeneca, outside the submitted work. She has also received a grant from the Dept of Veterans Affairs.

Support statement: This study was funded by the Dept of Veterans Affairs, Rehabilitation Research and Development Service (Career Development Award F6847W (M.L. Moy), Merit Award O1150 (M.L. Moy) and Career Development Award 2 IK2 RX002165 (E.S. Wan)). Funding information for this article has been deposited with the Crossref Funder Registry.

\section{References}

1 Global Initiative for Chronic Obstructive Lung Disease. Global Strategy for the Diagnosis, Management, and Prevention of Chronic Obstructive Pulmonary Disease. 2017. http://goldcopd.org/gold-2017-global-strategydiagnosis-management-prevention-copd Date last accessed: June 1, 2018. 
2 Moy ML, Teylan M, Weston NA, et al. Daily step count predicts acute exacerbations in a US cohort with COPD. PLoS One 2013; 8: e60400.

3 Nguyen HQ, Chu L, Amy Liu IL, et al. Associations between physical activity and 30-day readmission risk in chronic obstructive pulmonary disease. Ann Am Thorac Soc 2014; 11: 695-705.

4 Moy ML, Gould MK, Liu IA, et al. Physical activity assessed in routine care predicts mortality after a COPD hospitalisation. ERJ Open Res 2016; 2: 00062-2015.

5 Waschki B, Kirsten A, Holz O, et al. Physical activity is the strongest predictor of all-cause mortality in patients with COPD: a prospective cohort study. Chest 2011; 140: 331-342.

6 Moy ML, Teylan M, Weston NA, et al. Daily step count is associated with plasma C-reactive protein and IL-6 in a US cohort with COPD. Chest 2014; 145: 542-550.

7 Fuertes E, Carsin AE, Antó JM, et al. Leisure-time vigorous physical activity is associated with better lung function: the prospective ECRHS study. Thorax 2018; 73: 376-384.

8 Demeyer H, Costilla-Frias M, Louvaris Z, et al. Both moderate and severe exacerbations accelerate physical activity decline in COPD patients. Eur Respir J 2018; 51: 1702110.

9 Gimeno-Santos E, Frei A, Steurer-Stey C, et al. Determinants and outcomes of physical activity in patients with COPD: a systematic review. Thorax 2014; 69: 731-739.

10 Coultas DB, Jackson BE, Russo R, et al. Home-based physical activity coaching, physical activity, and health care utilization in chronic obstructive pulmonary disease. Chronic Obstructive Pulmonary Disease Self-Management Activation Research Trial secondary outcomes. Ann Am Thorac Soc 2018; 15: 470-478.

11 Mendoza L, Horta P, Espinoza J, et al. Pedometers to enhance physical activity in COPD: a randomised controlled trial. Eur Respir J 2015; 45: 347-354.

12 Moy ML, Collins RJ, Martinez $\mathrm{CH}$, et al. An internet-mediated pedometer-based program improves health-related quality-of-life domains and daily step counts in COPD: a randomized controlled trial. Chest 2015; 148: 128-137.

13 Demeyer H, Louvaris Z, Frei A, et al. Physical activity is increased by a 12-week semiautomated telecoaching programme in patients with COPD: a multicentre randomised controlled trial. Thorax 2017; 72: 415-423.

14 Wan ES, Kantorowski A, Homsy D, et al. Promoting physical activity in COPD: insights from a randomized trial of a web-based intervention and pedometer use. Respir Med 2017; 130: 102-110.

15 Mantoani LC, Rubio N, McKinstry B, et al. Interventions to modify physical activity in patients with COPD: a systematic review. Eur Respir J 2016; 48: 69-81.

16 Martinez CH, Moy ML, Nguyen HQ, et al. Taking Healthy Steps: rationale, design and baseline characteristics of a randomized trial of a pedometer-based Internet-mediated walking program in veterans with chronic obstructive pulmonary disease. BMC Pulm Med 2014; 14: 12.

17 Moy ML, Martinez CH, Kadri R, et al. Long-term effects of an internet-mediated pedometer-based walking program for chronic obstructive pulmonary disease: randomized controlled trial. J Med Internet Res 2016; 18: e215.

18 Moy ML, Janney AW, Nguyen HQ, et al. Use of pedometer and Internet-mediated walking program in patients with chronic obstructive pulmonary disease. J Rehabil Res Dev 2010; 47: 485-496.

19 Miller MR, Hankinson J, Brusasco V, et al. Standardisation of spirometry. Eur Respir J 2005; 26: 319-338.

20 ATS Committee on Proficiency Standards for Clinical Pulmonary Function Laboratories. ATS statement: guidelines for the six-minute walk test. Am J Respir Crit Care Med 2002; 166: 111-117.

21 Jones PW, Quirk FH, Baveystock CM, et al. A self-complete measure of health status for chronic airflow limitation. The St. George's Respiratory Questionnaire. Am Rev Respir Dis 1992; 145: 1321-1327.

22 Bestall JC, Paul EA, Garrod R, et al. Usefulness of the Medical Research Council (MRC) dyspnoea scale as a measure of disability in patients with chronic obstructive pulmonary disease. Thorax 1999; 54: 581-586.

23 Phan T, Carter O, Adams C, et al. Discriminant validity of the Hospital Anxiety and Depression Scale, Beck Depression Inventory (II) and Beck Anxiety Inventory to confirmed clinical diagnosis of depression and anxiety in patients with chronic obstructive pulmonary disease. Chron Respir Dis 2016; 13: 220-228.

24 Sherbourne CD, Stewart AL. The MOS social support survey. Soc Sci Med 1991; 32: 705-714.

25 Danilack VA, Okunbor O, Richardson CR, et al. Performance of a pedometer to measure physical activity in a U.S. cohort with chronic obstructive pulmonary disease. J Rehabil Res Dev 2015; 52: 333-342.

26 Matthews CE, Hagstromer M, Pober DM, et al. Best practices for using physical activity monitors in population-based research. Med Sci Sports Exerc 2012; 44: S68-S76.

27 Moy ML, Danilack VA, Weston NA, et al. Daily step counts in a US cohort with COPD. Respir Med 2012; 106 962-969.

28 Hankinson JL, Odencrantz JR, Fedan KB. Spirometric reference values from a sample of the general U.S population. Am J Respir Crit Care Med 1999; 159: 179-187.

29 Nguyen HQ, Fan VS, Herting J, et al. Patients with COPD with higher levels of anxiety are more physically active. Chest 2013; 144: 145-151.

30 Miravitlles M, Cantoni J, Naberan K. Factors associated with a low level of physical activity in patients with chronic obstructive pulmonary disease. Lung 2014; 192: 259-265.

31 Dueñas-Espín I, Demeyer H, Gimeno-Santos E, et al. Depression symptoms reduce physical activity in COPD patients: a prospective multicenter study. Int J Chron Obstruct Pulmon Dis 2016; 11: 1287-1295.

32 Shankar A, McMunn A, Demakakos P, et al. Social isolation and loneliness: prospective associations with functional status in older adults. Health Psychol 2017; 36: 179-187.

33 Kew KM, Mavergames C, Walters JA. Long-acting beta2-agonists for chronic obstructive pulmonary disease. Cochrane Database Syst Rev 2013; 10: CD010177.

34 Barr RG, Bourbeau J, Camargo CA, et al. Inhaled tiotropium for stable chronic obstructive pulmonary disease. Cochrane Database Syst Rev 2005; 2: CD002876.

35 Antonelli A, Torchio R, Bertolaccini L, et al. Contribution of $\beta$-adrenergic receptors to exercise-induced bronchodilatation in healthy humans. Respir Physiol Neurobiol 2012; 184: 55-59.

36 Redelmeier DA, Bayoumi AM, Goldstein RS, et al. Interpreting small differences in functional status: the six minute walk test in chronic lung disease patients. Am J Respir Crit Care Med 1997; 155: 1278-1282.

37 Polkey MI, Spruit MA, Edwards LD, et al. Six-minute-walk test in chronic obstructive pulmonary disease: minimal clinically important difference for death or hospitalization. Am J Respir Crit Care Med 2013; 187: 382-386. 
Hamer M, Sabia S, Batty GD, et al. Physical activity and inflammatory markers over 10 years. Follow-up in men and women from the Whitehall II cohort study. Circulation 2012; 126: 928-933.

39 Danilack VA, Weston NA, Richardson CR, et al. Reasons persons with COPD do not walk and relationship with daily step count. COPD 2014; 11: 290-299.

40 Demeyer H, Burtin C, Hornikx M, et al. The minimal important difference in physical activity in patients with COPD. PLoS One 2016; 11: e0154587.

41 Singh SJ, Puhan MA, Andrianopoulos V, et al. An official systematic review of the European Respiratory Society/ American Thoracic Society: measurement properties of field walking tests in chronic respiratory disease. Eur Respir J 2014; 44: 1447-1478.

42 Holland AE, Spruit MA, Troosters T, et al. An official European Respiratory Society/American Thoracic Society technical standard: field walking tests in chronic respiratory disease. Eur Respir J 2014; 44: 1428-1446.

43 Sciurba F, Criner GJ, Lee SM, et al. Six-minute walk distance in chronic obstructive pulmonary disease: reproducibility and effect of walking course layout and length. Am J Respir Crit Care Med 2003; 167: 1522-1527.

44 Hernandes NA, Wouters EF, Meijer K, et al. Reproducibility of 6-minute walking test in patients with COPD. Eur Respir J 2011; 38: 261-267. 